\begin{tabular}{|c|l|}
\hline Title & Time domain Brillouin scattering assisted by diffraction gratings \\
\hline Author(s) & Matsuda, Osamu; Pezeril, Thomas; Chaban, levgeniia; Fujita, Kentaro; Gusev, Vitalyi \\
\hline Citation & $\begin{array}{l}\text { Physical Review B, 97(6), 064301 } \\
\text { https://doi.org/10.1103/PhysRevB.97.064301 }\end{array}$ \\
\hline Issue Date & 2018.02-05 \\
\hline Doc URL & http://hdl.handle.net/2115/68491 \\
\hline Rights & @2018A American Physical Society \\
\hline Type & article \\
\hline File Information & PhysRevB.97.064301.pdf \\
\hline
\end{tabular}

Instructions for use 


\title{
Time-domain Brillouin scattering assisted by diffraction gratings
}

\author{
Osamu Matsuda, ${ }^{1, *}$ Thomas Pezerill, ${ }^{2}$ Ievgeniia Chaban, ${ }^{2}$ Kentaro Fujita, ${ }^{1}$ and Vitalyi Gusev ${ }^{3, \dagger}$ \\ ${ }^{1}$ Division of Applied Physics, Faculty of Engineering, Hokkaido University, 060-8628 Sapporo, Japan \\ ${ }^{2}$ Institut Molécules et Matériaux du Mans, UMR CNRS 6283, Le Mans Université, 72085 Le Mans, France \\ ${ }^{3}$ Laboratoire d'Acoustique de l'Université du Mans, UMR CNRS 6613, Le Mans Université, Av. O. Messiaen, 72085 Le Mans, France
}

(Received 1 May 2017; revised manuscript received 25 November 2017; published 5 February 2018)

\begin{abstract}
Absorption of ultrashort laser pulses in a metallic grating deposited on a transparent sample launches coherent compression/dilatation acoustic pulses in directions of different orders of acoustic diffraction. Their propagation is detected by delayed laser pulses, which are also diffracted by the metallic grating, through the measurement of the transient intensity change of the first-order diffracted light. The obtained data contain multiple frequency components, which are interpreted by considering all possible angles for the Brillouin scattering of light achieved through multiplexing of the propagation directions of light and coherent sound by the metallic grating. The emitted acoustic field can be equivalently presented as a superposition of plane inhomogeneous acoustic waves, which constitute an acoustic diffraction grating for the probe light. Thus the obtained results can also be interpreted as a consequence of probe light diffraction by both metallic and acoustic gratings. The realized scheme of time-domain Brillouin scattering with metallic gratings operating in reflection mode provides access to wide range of acoustic frequencies from minimal to maximal possible values in a single experimental optical configuration for the directions of probe light incidence and scattered light detection. This is achieved by monitoring the backward and forward Brillouin scattering processes in parallel. Potential applications include measurements of the acoustic dispersion, simultaneous determination of sound velocity and optical refractive index, and evaluation of samples with a single direction of possible optical access.
\end{abstract}

DOI: 10.1103/PhysRevB.97.064301

\section{INTRODUCTION}

Picosecond acoustic interferometry (PAI) is a powerful opto-acousto-optic technique for nondestructive and noncontact testing of transparent materials at the nanoscale [1-13]. First, using an ultrashort pump laser pulse, a propagating picosecond coherent acoustic pulse (CAP) is launched into the material. Second, partial scattering of a continuously timedelayed ultrashort probe laser pulse by the launched CAP is used to monitor the propagation of this nanometers scale acoustic perturbation through the material. The weak light pulses scattered by the CAP interfere at the photodetector with the probe light pulses of significantly higher amplitude reflected from various interfaces of the sample, such as the interfaces of the tested material with air and with the optoacoustic transducer, for example. The signal of the transient optical reflectivity is proportional, in leading order, to the product of the amplitudes of these two scattered light fields. Thus, heterodyning of a weak field against a strong one is achieved. The detected signal of time-resolved optical reflectivity in this so-called pump-probe scheme contains a sinusoidal oscillating component whose physical origin is the Brillouin scattering (BS) of the probe light by the CAP. The frequency of this oscillation depends on the angle between the propagation directions of the probe light and that of the coherent acoustic waves. It is precisely equal to the shift

\footnotetext{
*omatsuda@eng.hokudai.ac.jp

†Vitali.Goussev@univ-lemans.fr
}

in frequency of the scattered light that would be caused by thermal phonons propagating in the same direction as the CAP and could be resolved using optical spectrometers in classic frequency-domain BS (FDBS) experiments [14-17]. That is why PAI is also often called time-domain Brillouin scattering (TDBS).

An important limitation of TDBS in comparison with FDBS is that a significantly narrower part of the acoustic spectra is accessible by TDBS. In FDBS, by varying the angle between the directions in which the probe light is incident and in which the scattered light is detected, the direction of the thermal phonons for testing can be selected $[14,15,17]$. Because thermal phonons are available in all directions, this approach is very flexible in providing the opportunity to significantly vary the angle between the directions of the probe light and that of the phonon wave vectors. Thermal phonons of highest frequency are accessible in the so-called backscattering configuration [14,17], when the probe light is scattered by the counter-propagating or co-propagating phonons (annihilation or creation of phonons, respectively). Thermal phonons of the lowest frequency are detectable in the forward scattering configuration when the probe light and the phonons are propagating along nearly orthogonal directions, as in the so-called platelet configuration [17]. In TDBS, the situation is very different. In common TDBS experiments, the lateral dimensions of the optoacoustic generators are controlled by the size of the laser pump focus and typically significantly exceed the spatial lengths of the CAP emitted by them in the materials. Thus the diffraction length of the emitted CAP in typical TDBS experiments significantly exceeds its 
attenuation length, while the direction of the CAP is mostly fixed to quasiperpendicular to the sample surface illuminated by the pump laser pulse. Then the only possibility to vary the frequency of the tested phonon is to change the direction of the probe light propagation relative to the fixed direction of the CAP propagation. However, most of the TDBS applications are intended for the diagnostics of either thin coatings/multilayers deposited on bulk samples or thin plates/membranes with an optical access to the launched CAP only through the surfaces normal to CAP propagation direction. For simplicity, we will think about an isotropic medium throughout the paper, unless otherwise stated. The maximum angle of the probe light transmitted through the air/material surface relative to the direction of CAP propagation is theoretically $\theta_{i}^{\max }=\arcsin (1 / n)$, where $n$ is the refractive index of the material at the probe wavelength $\lambda$, when in air the light is skimming along the surface. Thus, for $n>\sqrt{2}$, the angle is smaller than $45^{\circ}$. In the resulting backscattering-type configuration, the frequency of the phonon detected by TDBS is [18-20]

$$
f_{B}=\frac{2 v n}{\lambda} \sqrt{1-\sin ^{2}\left(\theta_{i}\right)}=\frac{2 v n}{\lambda} \sqrt{1-\sin ^{2}(\theta) / n^{2}},
$$

where $v$ is the longitudinal sound velocity of the material and $\theta$ is the angle of incidence in air. Thus, for $n>\sqrt{2}$, even the lowest frequency theoretically accessible by TDBS, $f_{B}^{\min }$, is close to the maximum one, $f_{B}^{\min }>f_{B}^{\max } / \sqrt{2} \simeq 0.71 f_{B}^{\max }$. For more convenient but still large angle of incidence, $\theta=$ $60^{\circ}$, the estimate is $f_{B}^{\min }>\sqrt{5 / 8} f_{B}^{\max } \simeq 0.79 f_{B}^{\max }$. Thus, the tunability of the Brillouin frequency expressed in Eq. (1) is limited by refraction at the air/sample interface. This indicates the limitations in application of TDBS for revealing and identifying the frequency dispersion of material properties such as, for example, sound velocity and attenuation. Another limitation of typical TDBS scheme is that, in accordance with Eq. (1), when fixing the external angle of probe incidence and measuring the Brillouin frequency $(\mathrm{BF}), f_{B}$, we get information on the combination of two material parameters ( $v$ and $n$ ). Then, to determine them independently, TDBS measurements should be conducted at least at two different angles $\theta$ [19-21], while in FDBS there exists an experimental forward scattering configuration, which is called platelet configuration [17], providing the opportunity to measure sound velocity by a single measurement without determining the optical refractive index.

Below we propose a TDBS experiment, using gratings consisting of periodically arranged pump light absorbing parallel rods, that gives access to larger angles BS, including those of forward-type scattering, and, as a consequence, broadens the spectrum of the detectable/accessible phonons and also provides opportunity to measure sound velocity within a single mutual orientation of the optical excitation and detection. The rods could be metallic, for example, as in our experiments (Fig. 1). When a grating with a period $p$ is used for the generation of the CAPs instead of a metallic thin film, which is typically used for this purpose, the coherent acoustic waves will be emitted not only normally to the plane of the gratings but also in all directions corresponding to the possible nonzeroth diffraction orders of the acoustic waves by this grating, thus, multiplexing the propagation directions of CAPs in the sample. Additionally, the grating can diffract both transmitted

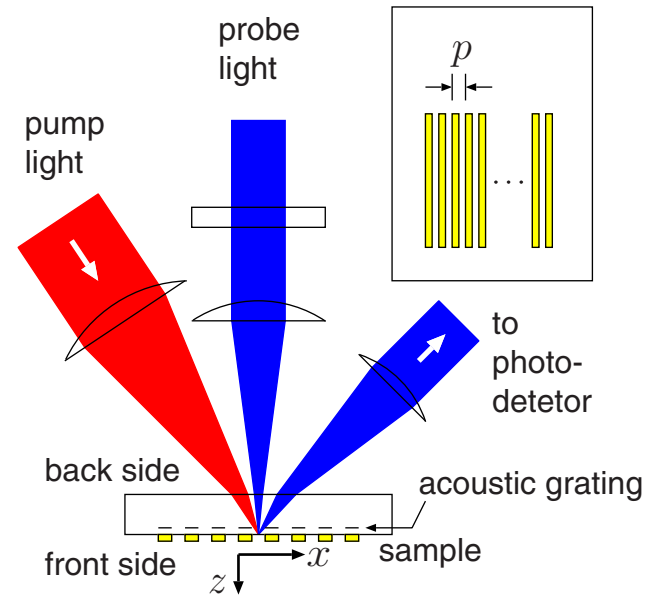

FIG. 1. The optical setup for the measurement. Both pump and probe beams are incident from the backside (without grating) of the sample. Signal is detected in the direction of the first order optical diffraction from the backside of the sample. Insert: Schematic diagram of the periodic gold rods formed on a fused silica substrate. The nominal period $p$ is 587.0 or $479.2 \mathrm{~nm}$, corresponding to first-order optical diffraction of the probe light in air at around $45^{\circ}$ and $60^{\circ}$, respectively. The grating spacing is significantly exaggerated: in reality several gold rods are accommodated within the laser spot so that the light is well diffracted.

and reflected probe light, thus, multiplexing the propagation directions of the probe laser pulses inside the sample. Both of these factors should potentially lead to an increase of the maximum angles between the propagation directions of the coherent sound and of the probe light, and give access for TDBS to forward-type photon scattering processes. Moreover, application of the grating should provide an opportunity to monitor simultaneously the same acoustic mode, for example, longitudinal, at different frequencies.

One may notice the similarity between the proposed method and the transient grating (TG) method [22-25] in which gratinglike acoustic waves are generated by two crossing light beams. Both methods are employing possible emission of coherent acoustic phonons along multiple different propagation directions. The most important difference of the proposed method with the TG method is similar to that with FDBS. For the same fixed directions of the probe light incidence and the scattered light detection, i.e., without modifying the optical scheme, our method provides opportunity to monitor acoustic waves propagating in larger number of different directions. This is achieved by using the metallic grating for multiplexing the directions of the probe light propagation before its Brillouin scattering and also after its Brillouin scattering. Multiple spectral peaks are observed by the proposed method even if a single acoustic mode (longitudinal in our experiments) is efficiently generated and detected. Both forward and backward Brillouin scattering processes are monitored in a single optical configuration.

It is also worth noting earlier reports on the deposition of the structured optoacoustic transducers on the sample surface for the emission of the diffracting picosecond acoustic fields $[26,27]$. The experiments were conducted not only with 
laterally periodic metallic structures [26] but, recently, even with a single metallic nanowire dropped on the sample surface [27]. However, the detection of the diffracted acoustic field was achieved only on its arrival at the opposite surface of the sample by a probe light incident normally on the surface. Our proposed scheme with multiplexing of probe light directions by metallic grating provides opportunity to probe inside optically transparent samples at multiple directions of light incidence the evolution of the picosecond acoustic field inside the material caused by its diffraction and attenuation. In comparison with probing diffracting acoustic beam by a collinearly propagating diffracting optical probe beam, achieved in Ref. [13], the proposed scheme provides information simultaneously on multiple frequencies of the picosecond acoustic field.

\section{EARLIER EXPERIMENTS WITH METALLIC GRATING DIFFRACTING PROBE LIGHT IN TRANSMISSION MODE}

After realizing these theoretical predictions experimentally in the schema presented in Fig. 1, we have found that a part of them could have been confirmed just through the dedicated analysis of the experimental results published much earlier in Ref. [18]. In this publication, the pump-probe optical schema was for the first time successfully applied to reveal the elastic motions of the metallic grating deposited on the sample surface (gold rods on fused silica, the same combination of materials as in our experiments). The principle difference in comparison with our optical experiments is in conducting pumping and probing of the samples from the grating side of the sample. The experimental setup was similar to Fig. 1 but the sample was placed upside down: both pump and probe light comes from the front side (with grating) of the sample. The title of this publication, "Study of vibrational modes of gold nanostructures by picosecond ultrasonics," and its abstract, both emphasize the successful identification of the low-lying frequencies in the transient reflectivity spectrum with the normal modes of the nanorods coupled to the substrate. Because of this fact and the years passed we forgot that in Ref. [18], three additional high-lying frequencies (modes I-III in Fig. 10 of Ref. [18]) have been detected and reproduced by the solutions of the theoretically formulated problem, although the origin of not all of them had been understood even qualitatively. The origin of mode I was identified with the backscattering-type process described in Eq. (1) without any influence of the grating on it as illustrated in Fig. 2(a). We presented the revealed ray path for the probe light monitoring mode II in Fig. 2(b). The origin of the mode II was related with the influence of the grating on the probe light field only, i.e., without accounting for the difference in the directivity patterns of the CAPs emitted by the metallic grating and a metallic thin film. Finally the origin of mode III was not understood. Based on our proposal that grating directs both the probe light and the generated acoustic waves in different orders of the diffraction, the interpretation of the physical origin of the modes II and III is straightforward. With the coordinate system presented in Fig. 1, the wave vector of the in-coming probe photon from air toward the sample is given by $\mathbf{k}=\left(k_{x}, 0, k_{z}\right)=\left(k_{x}, 0, \sqrt{k^{2}-k_{x}^{2}}\right)$, where $k=|\mathbf{k}|$. Then in transmission from air into the sample, the probe field is diffracted by the metallic grating in multiple directions defined
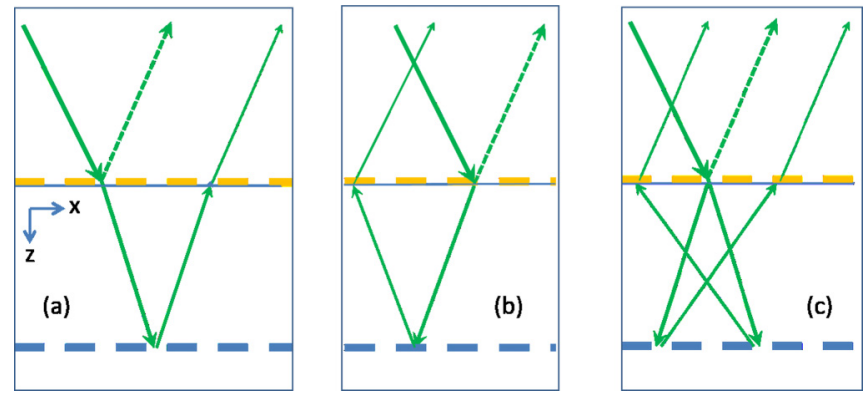

FIG. 2. Diagrams of the probe light ray paths corresponding to the detection of three modes of the Brillouin oscillations in the experimental scheme used in Ref. [18]. The metallic grating, presented by yellow bars, is on the surface of the transparent sample. The metallic grating operates in transmission mode. It launches a coherent acoustic field propagating downward, which is presented symbolically by blue grating. The dashed ray indicates the path of the strong scattered probe light beam, which is noninteracting with the acoustic waves and is used on photodetector for heterodyning of weak probe light beams scattered by the coherent acoustic waves. (a) Mode I of the oscillations is detected without probe light diffraction by the metallic grating and by the acoustic grating. The probe light exhibits refractions at the plane surface of the sample and reflection by the plane homogeneous in amplitude front of the CAP. (b) The detection of mode II of the oscillations is assisted by two first-order diffractions of the probe light by the metallic grating, one before and another after probe light reflection by the plane homogeneous in amplitude front of the CAP. (c) Mode III of the oscillations is detected as a result of two different probe light ray paths both containing one first order diffraction by metallic grating and one first order diffraction by the acoustic grating, although in different sequence. These illustrative drawings correspond to the experiments[18] with 600 -nm period grating.

by the wave vectors,

$$
\mathbf{k}_{i}=\left(k_{x}+m_{i} q, 0, \sqrt{k_{i}^{2}-\left(k_{x}+m_{i} q\right)^{2}}\right) .
$$

Here, $q=2 \pi / p$ is the grating wave number, $k_{i}=n k$ is the wave number of the probe photon in the sample, while $m_{i}=$ $0, \pm 1, \pm 2, \ldots$ indicates the order of the diffraction peak. The probe light field backscattered by the phonons should have the propagation directions described by

$$
\mathbf{k}_{s}=\left(k_{x}+m_{s} q, 0,-\sqrt{k_{s}^{2}-\left(k_{x}+m_{s} q\right)^{2}}\right),
$$

where $m_{s}=0, \pm 1, \pm 2, \ldots$ Only the light propagating along these directions, when transmitted from the sample into the air, could be diffracted by the grating in the detection direction, given in Ref. [18] by $\mathbf{k}_{d}=\left(k_{x}, 0,-\sqrt{k^{2}-k_{x}^{2}}\right)$. Note that the indexes $i$ and $s$ are introduced for the photon incident on the phonon and scattered by the phonon, respectively. These photons are propagative in the limited number of the diffraction orders defined by $k_{i, s}>\left|k_{x}+m_{i, s} q\right|$ and evanescent in the rest. The wave vector of the acoustic phonon participating in the $\mathrm{BS}$ is given by the law of the momentum conservation [14-17], $\mathbf{k}_{B}= \pm\left(\mathbf{k}_{s}-\mathbf{k}_{i}\right)$ where the plus sign corresponds to absorption of the acoustic phonon (anti-Stokes) and the minus sign to its emission (Stokes). Then the modulus of the wave vector of the coherent acoustic phonon which has participated 
in the $\mathrm{BS}$ is

$$
\begin{aligned}
k_{B}=\frac{2 \pi f_{B}}{v}= & \left\{\left[\left(m_{s}-m_{i}\right) q\right]^{2}+\left[d_{s} \sqrt{k_{i}^{2}-\left(k_{x}+m_{s} q\right)^{2}}\right.\right. \\
& \left.\left.-d_{i} \sqrt{k_{i}^{2}-\left(k_{x}+m_{i} q\right)^{2}}\right]^{2}\right\}^{1 / 2} .
\end{aligned}
$$

Here the difference in the frequencies of the incident and scattered photons is neglected, $k_{s}=k_{i}$, as usual, while the parameters $d_{s, i}= \pm 1$ are introduced by us to account for the variety of possible directions of incident and scattered light, in the general case. They take the value +1 or -1 according to the positive and negative values of the $z$ component, respectively, of $\mathbf{k}_{i}$ and $\mathbf{k}_{s}$. In the experiments in Ref. [18], $d_{i}=-d_{s}=1$ corresponds to monitoring of the backscattered light only, because the forward scattered light does not return to the detection region. The proposed Eq. (2) provides the frequency of the mode I as in Eq. (1) when $m_{s}=m_{i}=0$ [see Fig. 2(a)]. The proposed Eq. (2) reproduces Eq. (14) from Ref. [18] when $m_{s}=m_{i}=m$ and, thus, reveals the physical sense of the parameter $m$ in Ref. [18]. The derived condition also confirms the suggestion in Ref. [18] that in the mode II the light is scattered by the plane acoustic front propagating in the direction normal to the surface. In fact, the projection of the phonon wave vector along the surface (along the $x$ axis) is equal to the difference between the projections of the photons wave vectors, $\left(m_{s}-m_{i}\right) q$, and, thus, for the revealed $m_{s}=m_{i}=m$ it is equal to zero. The phonons, which scatter light in mode II, propagate normally to the surface. Moreover the experimentally observed mode II corresponds to $|m|=1, \operatorname{sign}(m)=-\operatorname{sign}\left(k_{x}\right)$. We presented the revealed ray path for the probe light monitoring mode II in Fig. 2(b). So the origin of the mode II is the scattering by the CAP of the light directed by the metallic grating in such diffraction order whose direction is the closest to the CAP propagation direction. Finally, Eq. (2) attributes the origin of the mode III to the following two degenerate in frequency processes. In the first one the metallic grating directs the incident light like in mode II, i.e., in the diffraction order closest to the surface normal, but then the coherent acoustic wave scatters light in such direction towards the front surface of the sample, from which it can be detected without additional diffraction $\left(\left|m_{i}\right|=1, \operatorname{sign}\left(m_{i}\right)=-\operatorname{sign}\left(k_{x}\right), m_{s}=0\right)$. In the second one, the acoustic wave backscatters nondiffracted (zeroth order) probe light in the diffraction order of light closest to the surface normal, from which it is diffracted to the detection direction by the optical grating in transmission from the sample into the air $\left[m_{i}=0,\left|m_{s}\right|=1, \operatorname{sign}\left(m_{s}\right)=-\operatorname{sign}\left(k_{x}\right)\right]$. We presented the revealed rays paths for the probe light monitoring mode III in Fig. 2(c). In both of these processes the acoustic waves are not just reflecting the incident light like the mirrors but are modifying the direction of the scattered light relative to one predicted by the Snell's law. One can say that the acoustic waves are diffracting the incident light and are functioning as diffraction gratings with the wave number $q$. It is natural in the following to use for the acoustic field, generated in the sample by pump laser pulses incident on the metallic grating, the term acoustic grating because it is periodic along the $x$ axis. Note that this terminology was used already for example for the description of the acoustic waves generated by laser induced gratings, i.e., by the intensity interference patterns that can be created by two light beams incident on the sample surface at angles $\theta$ and $-\theta$ [28-31]. It was demonstrated that the acoustic field emitted by the pulsed laser gratings can be decomposed into the so-called plane inhomogeneous acoustic modes [28-31], i.e., the acoustic waves with plane phase fronts parallel to the sample surface but modulated in amplitude with the pattern of the laser intensity grating. Thus these modes can be naturally called acoustic gratings. In the experiments in Ref. [18] and in our experiments presented below in Sec. III (Fig. 1) the acoustic gratings are generated by the metallic gratings. Their ability not just to reflect/transmit but to diffract the incident light is due to their amplitude periodic modulation. Using the suggested terminology, all the high-lying frequency modes detected in Ref. [18] can be explained by processes involving only two diffractions of probe light by the gratings, i.e., either two diffractions by metallic grating (mode II) or one diffraction by the metallic grating plus one diffraction by the acoustic grating (mode III). Although in these experiments only the backscattering-type processes were observed and only those involving first diffraction orders, they are in favor of our proposal formulated in Introduction that, in the experiments with gratings, multiple frequencies corresponding to BS processes with different angles between sound and light propagation directions can be detected simultaneously.

\section{EXPERIMENTS WITH METALLIC GRATING DIFFRACTING PROBE LIGHT IN REFLECTION MODE}

In our TDBS experiments conducted from the opposite side of the sample (Fig. 1) than in Ref. [18], we have additionally detected the BFs corresponding to forward scattering processes, to the processes involving three consecutive diffractions of probe light (two by metallic grating and one by acoustic grating) and also to the processes involving light from the higher diffraction orders than the first order. The gold gratings are made by the electron beam lithography and liftoff technique on a fused silica substrate of $1-\mathrm{mm}$ thickness. The insert in Fig. 1 shows a schematic structure of the sample. Two samples with different nominal grating periods, $p=587.0$ and $479.2 \mathrm{~nm}$, are prepared. We refer the former sample as $45^{\circ}$, whereas the latter as $60^{\circ}$, because they are prepared to have the first-order diffraction peaks near these directions for the reflected probe light incident normally at the grating. The designed width of gold rod/gap is $300 \mathrm{~nm} / 287 \mathrm{~nm}$ for the $45^{\circ}$ sample and $250 \mathrm{~nm} / 229 \mathrm{~nm}$ for the $60^{\circ}$ sample. The actual width of the gold rod (gap) becomes somewhat larger (smaller) than these designed values during the fabrication process. The thickness of the gold rods is about $50 \mathrm{~nm}$. A 2-nm-thick $\mathrm{Cr}$ layer is formed between the $\mathrm{SiO}_{2}$ substrate and the Au film to improve the film adhesion.

A standard laser picosecond setup is used. A mode-locked Ti:sapphire laser with a regenerative amplifier is used as the light source. The pulse width is $\sim 100 \mathrm{fs}$ and the repetition frequency is $260 \mathrm{kHz}$. Fundamental light pulses with a central wavelength of $800 \mathrm{~nm}$ are focused to the grating structure from the back side (the side without grating) of the sample (Fig. 1). The pulse energy is $80 \mathrm{~nJ} /$ pulse and the diameter of the focused region is $100 \mu \mathrm{m}$, covering nearly completely the rectangular grating area, whose lateral dimensions are similar $(100 \mu \mathrm{m}$ by $100 \mu \mathrm{m})$. The absorption of pump laser pulses in the metallic 

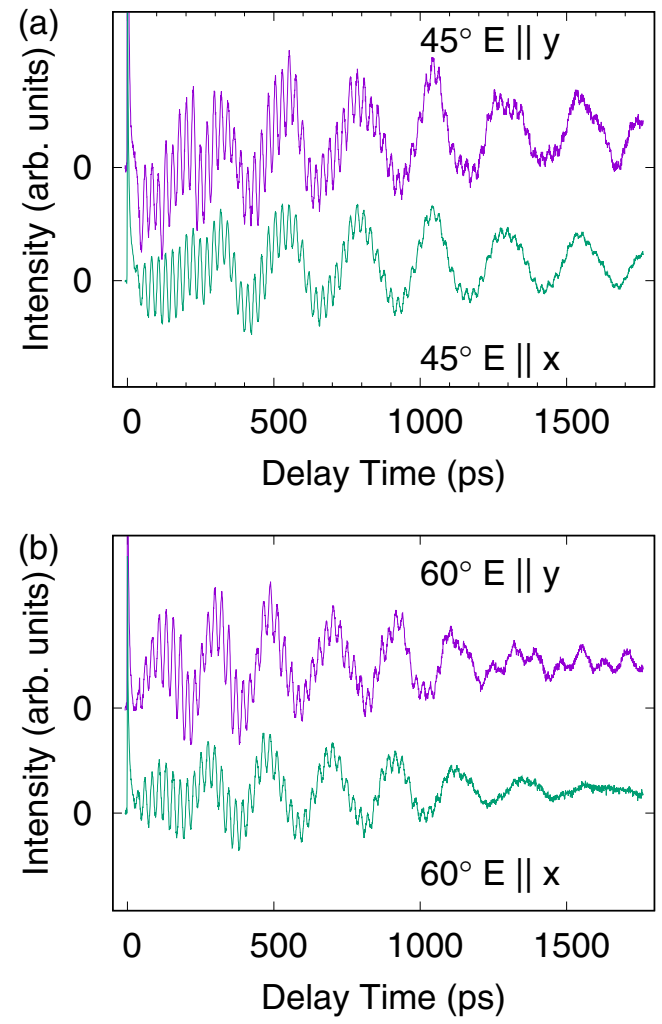

FIG. 3. Transient intensity variation of the first-order diffracted light as a function of the delay time between the pump and probe light pulses arrival at the sample. (a) For $45^{\circ}$ sample, and (b) for $60^{\circ}$ sample. The polarization of the incident probe light is indicated on the figure.

grating generates acoustic waves propagating in the sample along different orders of acoustic diffraction, as it is described in Introduction. Alternatively, the launched acoustic field could be viewed as inhomogeneous plane waves or acoustic grating propagating normally to the sample surface [28-31]. The acoustic grating propagating normal to the metallic grating is the result of the interference of the acoustic waves propagating along positive and negative orders of the acoustic diffraction.

The frequency doubled light pulses with a central wavelength of $400 \mathrm{~nm}$ are focused onto the grating structure from the back side at normal incidence. The pulse energy is $4 \mathrm{~nJ} /$ pulse and the diameter of the focused region is $10 \mu \mathrm{m}$. The probe light scattered by the whole structure in the first order of diffraction is fed to the photo detector to detect the light intensity modulation caused by the acoustic waves (Fig. 1). The polarization of the incident probe light is in parallel with the grating period ( $x$ axis, $\mathbf{E} \| \mathbf{x}$ ) or in parallel with the gold rods ( $y$ axis, $\mathbf{E} \| \mathbf{y}$ ). Figure 3 shows the raw data of the transient intensity variation of the diffracted light for the $45^{\circ}$ and $60^{\circ}$ samples with two probe polarizations $\mathbf{E} \| \mathbf{x}$ and $\mathbf{E} \| \mathbf{y}$. The delay time is the time between the pump and probe light pulses arrival at the sample.

Each transient curve consists of two major contributions: a slow oscillating component with a period around $200 \mathrm{ps,}$ and a fast oscillating component with a period around 20 ps. They resemble the curves presented in Fig. 2 of Ref. [18]. This indicates a possible splitting of the photo-induced motion
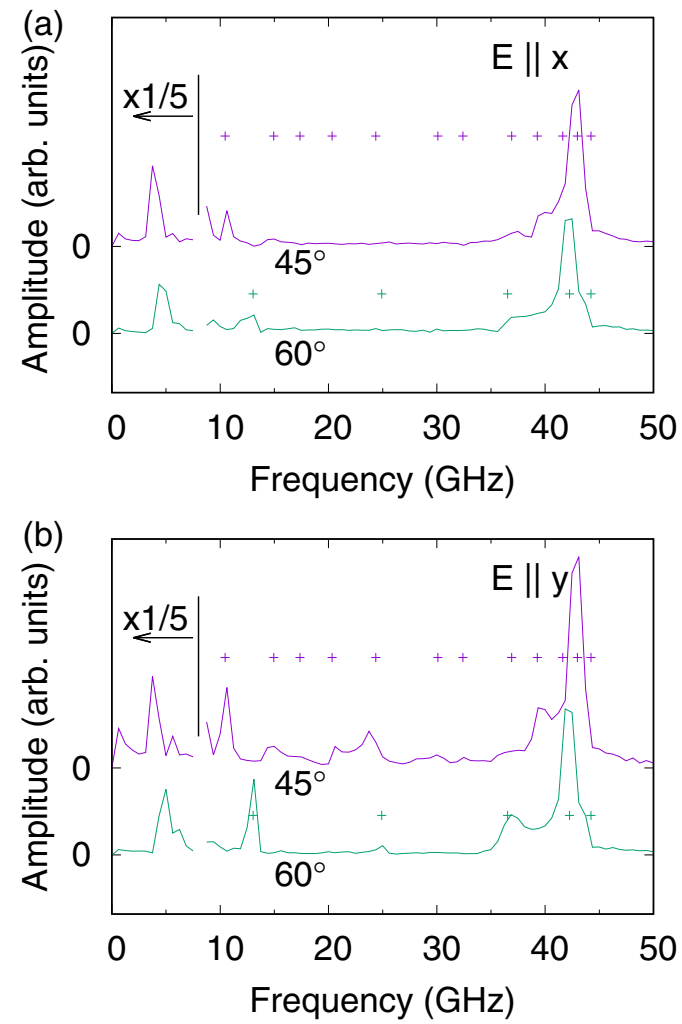

FIG. 4. The norm of Fourier amplitude of the data shown in Fig. 2. (a) For $\mathbf{E} \| \mathbf{x}$ and (b) for $\mathbf{E} \| \mathbf{y}$. The "+" symbols denote the theoretically calculated frequencies of all possible Brillouin scattering configurations with the fitted values for $n$ and $v$. For convenience of presentation, the amplitudes in the low-lying part of the spectra $(<8 \mathrm{GHz})$ are five times diminished.

of the sample into low-lying frequency modes and high-lying frequency modes as in Ref. [18]. To get further understanding on the origin of these oscillations, the obtained temporal signal is Fourier transformed with respect to the delay time. Figure 4 shows the norm of the Fourier amplitude as a function of frequency.

In the low-lying part of the spectrum below $8 \mathrm{GHz}$, where the amplitudes in Fig. 4 are intentionally diminished, we detect, similar to Ref. [18], up to three different modes. Following Ref. [18], we attribute them to the vibrations of the gold rods on the sample surface. Although these oscillations are beyond our interest here, it was straightforward to associate the strongest of the low-lying modes (at 3.8 and $5.0 \mathrm{GHz}$ in the $45^{\circ}$ and $60^{\circ}$ samples, respectively) with the L (longitudinal) mode defined in Ref. [18]. This was achieved using the results of numerical calculations of the resonance frequency of the rods with different widths and thicknesses presented in Fig. 8 of Ref. [18] and accounting for the fact that the frequency of the strongest mode in our experiments scales approximately inversely proportional to its width.

High-lying parts of the observed spectra in our experiments are always containing larger number of frequency peaks in comparison with Ref. [18]. For example, in $45^{\circ}$ sample in case of $\mathbf{E} \| \mathbf{y}$ probe polarization, the number of the frequencies that can be identified straightforwardly is nine [see Fig. 4(b) and Table I], i.e., at least three times more than that in Ref. [18]. 
TABLE I. Calculated (calc.) and experimental (exp.) frequencies of phonons involved in each Brillouin scattering configuration (config.) in which $m$ and $d$ in the notation $(m, d)$ give the diffraction order and the sign of the $z$ component of the light wave vector, respectively. The frequency value in the parentheses indicates that the corresponding peak is substantially broadened. The dash symbol $(-)$ indicates that the corresponding peak is not observed. The asterisk symbol (*) indicates that the expected peak is indistinguishable from the neighboring peak. The value in the square brackets indicates the corresponding peak just appears as a shoulder of the neighboring peak. The lines with empty values indicate that the mentioned configuration is degenerated to the one with values below. NA means the corresponding scattering mode involves evanescent photons. The thick horizontal line in the middle separates the upper part, where the data can be explained by the scattering processes involving probe light of the zeroth and the first orders of diffraction, from the lower part, where all the processes involve additionally the photons from the second diffraction order. Frequency unit: GHz.

\begin{tabular}{lcccccc}
\hline \hline & $\begin{array}{c}45^{\circ} \\
\text { calc. }\end{array}$ & $\begin{array}{c}45^{\circ} \\
\text { exp. } \\
(\mathbf{E} \| \mathbf{y})\end{array}$ & $\begin{array}{c}45^{\circ} \\
\text { exp. }\end{array}$ & $\begin{array}{c}60^{\circ} \\
\text { calc. }\end{array}$ & $\begin{array}{c}60^{\circ} \\
\text { exp. }\end{array}$ & $\begin{array}{c}60^{\circ} \\
\text { exp. } \| \mathbf{y})\end{array}$ \\
config. $\| \mathbf{x})$
\end{tabular}

All the experimentally observed modes in the high-lying part of the spectrum can be attributed to particular BS processes theoretically contributing to Eq. (2), when accounting for the differences between our experimental configuration and samples, and those in Ref. [18]. The first and the most important advantage of optically probing the sample from the back side (Fig. 1) consists in the additional opportunity to monitor forward-type BS processes by TDBS. This opportunity is related to the fact that in our experiments the metallic grating acts on the probe light in the reflection mode while in the optical schema of Ref. [18], i.e., when the sample in Fig. 1 is placed upside down and probed from the front side, the metallic grating acts on the probe light in the transmission mode only. This can be qualitatively understood considering four types of probe light scattering sequences which are contributing to the reflection of light by the sample in the direction towards
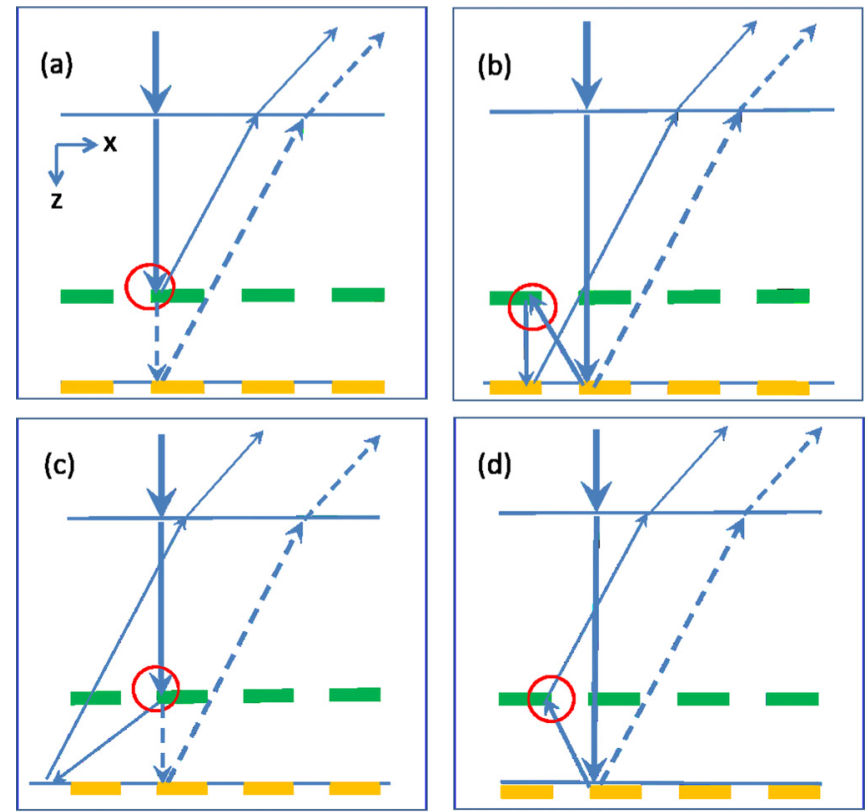

FIG. 5. Diagrams of the probe light ray paths illustrating four different sequences of light scattering in our experimental scheme presented in Fig. 1. The metallic grating, depicted as yellow bars, is on the lower surface of the transparent substrate. It operates in reflection mode. It launches a coherent acoustic field propagating upward, which is presented symbolically by a green grating. The dashed rays indicate the path of the strong scattered probe light beam, noninteracting with the acoustic waves, which is used on the photodetector for heterodyning of the weak probe light beams scattered by the coherent acoustic field. The red circles symbolically indicate the points of probe light scattering by the acoustic field, i.e., of the Brillouin scattering. (a) The probe light in the backward scattering process is directed by the acoustic grating into the first diffraction order towards the detector. (b) Backward scattering of the probe light by the acoustic grating between its two diffractions by the metallic grating. The second reflection/diffraction of the probe light by metallic grating directs it towards the detector. (c) Forward scattering by the acoustic grating of the probe light which subsequently is directed towards the detector by the metallic grating. (d) The probe light is first reflected/diffracted by the metallic grating and then followed in the direction of the detector due to its forward scattering by the acoustic grating. Note that in these illustrative drawings not all relevant ray paths are presented. The distance of the acoustic grating from the laser-irradiated top surface of the substrate is artificially reduced not in scale with the other dimensions for the compactness of the presentation. The illustrations correspond to our $45^{\circ}$ sample.

the detector. Accounting for the fact that the scattering of light by the acoustic grating is much weaker than that by the metallic one, only the sequences with a single scattering by the acoustic grating should be considered. The first and the simplest sequence is the diffraction of the probe light normally incident on the acoustic grating in reflection/backscattering mode towards the detector. This process corresponds to $k_{x}=0$, $d_{i}=-d_{s}=1, m_{i}=0$, and $m_{s}=1$ in Eq. (2), and is illustrated by the probe light ray diagram in Fig. 5(a). We remind here that the parameters $m_{i, s}$ numerate the diffraction orders, while $d_{i, s}$ fix the direction of the probe light propagation along the $z$ axis. The detection direction in our experiments 
is always $d_{d}=-1, m_{d}=1$, which is not necessarily the same as $d_{s}$ and $m_{s}$ as shown below. The second sequence including backscattering of light by the acoustic grating contains [see the ray paths diagrams in Fig. 5(b)] (a) the transmission of the probe light through the acoustic grating without diffraction, (b) reflection or diffraction of the light by the metallic grating in the different orders of the diffraction, (c) reflection/backscattering of light by the acoustic grating in the different orders of the diffraction [the process with $k_{x}=$ $0, d_{i}=-d_{s}=-1$, and arbitrary $m_{i, s}$ in Eq. (2)], and (d) reflection or diffraction of the light by the metallic grating in the direction of its detection $d_{d}=-1$ and $m_{d}=1$. Two other sequences include forward scattering of light by the acoustic grating $\left(d_{s}=d_{i}\right)$. The third sequence consists of [see Fig. 5(c)] (a) forward scattering of the probe light normally incident on the acoustic grating in the different orders of the diffraction [the process with $k_{x}=0, d_{i}=d_{s}=1, m_{i}=0$, and arbitrary $m_{s}$ in Eq. (2)] and (b) reflection or diffraction of the light by the metallic grating in the first order, i.e., in the direction of its detection. The fourth sequence includes [see Fig. 5(d)] (a) transmission of the probe light through the acoustic grating without scattering, (b) reflection or diffraction of the light by metallic grating in the different orders of the diffraction, and (c) forward scattering/transmission of the light by the acoustic grating in the first order, i.e., in the direction of its detection [the process with $k_{x}=0, d_{i}=d_{s}=-1$, arbitrary $m_{i}$ and $m_{s}=1$ in Eq. (2)].

We have found that for the identification of all BFs detected in all four conducted experiments it is sufficient to account only for the propagating (i.e., nonevanescent) incident and scattered light fields in the processes defined by Eq. (2), although only the light directed towards the detector should be obligatory propagating (because of the large distance between the region of its diffractions/scatterings and the back surface of the sample). Although in the second of the above described sequences both incident and scattered light fields could be potentially evanescent, while in the third and the forth sequences the evanescent could be the scattered and the incident light, respectively, it was sufficient for us to account (in addition to the zeroth order of the diffraction) only for the first and the second orders of the diffracted light in the $45^{\circ}$ sample and for the first order of the diffracted light in the $60^{\circ}$ sample. With the known refractive index of the fused silica in our sample $n=1.47-1.48$ (see below) the light in all other diffraction orders is evanescent $\left(k_{i, s}<\left|k_{x}+m_{i, s} q\right|\right)$. Thus it is possible to explain all the observed BFs only by the BS processes with the momentum conservation diagrams presented in Fig. 6. In this figure and later on, the state of the incident photon (left column) is identified by $\left(m_{i}, d_{i}\right)$, while the state of the scattered photon (upper row) by $\left(m_{s}, d_{s}\right)$. Blue, green, and red arrows represent the wave vectors of the incident photon, of the scattered photon, and of the phonon, respectively.

The wave vector diagrams for the Brillouin scattering processes, corresponding to the four probe light transmission/reflection sequences described above, are presented in the upper left, lower right, upper-right, and lower-left parts of Fig. 6, respectively. They are separated by continuous black lines. Equation (2) (for $k_{x}=0$ ) provides an opportunity to calculate the frequencies of all scattering processes depicted in Fig. 6. The calculated BFs based on the fitting (details will

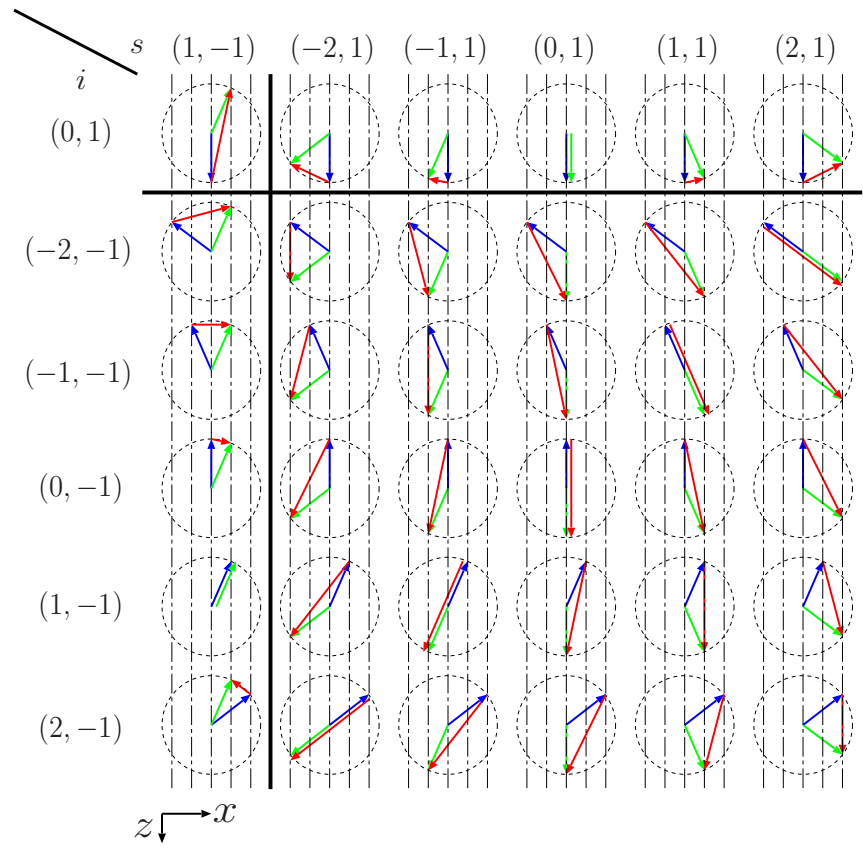

FIG. 6. The possible Brillouin scattering configurations for the normal incident probe light with the wave vector $\left(0,0, k_{i}\right)$ and the detection at the probe light along the first diffraction order with the wave vector $\left(q, 0,-\left(\left(k_{i}\right)^{2}-q^{2}\right)^{1 / 2}\right)$, both in the substrate. The wave vectors of photons before the scattering, $\mathbf{k}_{i}$, are shown in blue arrows. The wave vectors of the photons after the scattering, $\mathbf{k}_{s}$, are shown in green arrows. The wave vectors of the phonons, $\mathbf{k}_{B}$, are shown in red arrows. The $x$ and $z$ axes are shown at the bottom. In the left column, the state of the photon incident on the acoustic grating is identified by the diffraction order $m_{i}$ and the direction of its propagation along the $z$ axis, $d_{i}= \pm 1$, in the form $\left(m_{i}, d_{i}\right)$. In the upper row, the state of the photon scattered by the acoustic grating is identified by the diffraction order $m_{s}$ and the direction of its propagation along the $z$ axis, $d_{s}= \pm 1$, in the form $\left(m_{s}, d_{s}\right)$. The distance between the dash-dot vertical lines is equal to the wave number of the gratings, $q$. The vertical and horizontal continuous lines indicate the distinction of forward and backward scattering processes: the upper-left and lower-right processes involve the backward scattering, whereas the upper-right and lower-left processes involve the forward scattering.

be explained later) are presented in Table I together with the experimentally measured frequencies. For completeness, we list in the left column of Table I all the cases of degeneracy, i.e., when different scattering configurations in space correspond to the same phonon wave number. The notation "NA" in Table I marks the processes involving the photons from the second diffraction order in the $60^{\circ}$ sample, which are evanescent. It was not required to account for these evanescent processes to explain all of our experimental observations.

The theoretical estimates indicated in Table I are done with the following parameters of fused silica $n=1.482$ and $v=5.968 \times 10^{3} \mathrm{~m} / \mathrm{s}$, which are obtained by the least squares fit of the calculated frequencies to the experimentally obtained ones given in Table I except those in the square brackets. Note that the fitted $n$ and $v$ parameters are reasonably close to the literature values $n=1.471$ and $v=5968 \mathrm{~m} / \mathrm{s}$ [32]. The calculated values are also marked with "+" symbol in Fig. 4. 
The correspondence between the theoretically predicted and the measured Brillouin frequencies is fairly good.

\section{DISCUSSION}

We have already noted above that the larger number of Brillouin frequencies accessible in our experiments in comparison with those in Ref. [18] is due to the ability to monitor forward scattering processes in the configuration presented in Fig. 1. Another reason is in the larger number of the diffraction orders of the propagating probe light in our $45^{\circ}$ sample. From our calculation, the samples studied in Ref. [18] with 400 and $600 \mathrm{~nm}$ periodicity of the metallic gratings may involve the propagating photons in the zeroth and the first diffraction orders. Importantly, in our $45^{\circ}$ sample, the photons are propagative additionally in the second diffraction order. This increases the number of the efficient BS configurations as it could be appreciated from the lower part of Table I, where the theoretical interpretation of the detected BFs necessities the participation in the BS of the photons from the second diffraction order. The fact that the photons in the second diffraction order are propagating in the $45^{\circ}$ sample and are evanescent in the $60^{\circ}$ samples also explains our experimental observations that a larger number of the BFs are detected in the $45^{\circ}$ sample than in the $60^{\circ}$ sample (see Fig. 4 and Table I).

It was expected that the propagation of the probe light in the higher diffraction orders would require the participation in the BS processes of the phonons launched in the higher orders of diffraction in comparison with the earlier experiments. This is confirmed by our experimental results presented in Table I and explained by the diagrams in Fig. 6. Only in one of the processes, $(-1,-1) \rightarrow(1,-1)$, involving the first diffraction orders of light (dominating in the upper part of the Table I above the thick horizontal line in its center), the participation of the phonons of the second diffraction order, i.e., with the projection of the phonon wave vector on the $x$ axis equal to $\left|m_{s}-m_{i}\right| q=2 q$, is required to explain the particular experimentally detected frequency. Note that two other processes in the upper part of Table $\mathrm{I},( \pm 1,-1) \rightarrow$ $(\mp 1,1)$ and $( \pm 2,-1) \rightarrow(\mp 2,1)$, due to phonons emitted by the optoacoustic generator in the second and the fourth diffraction orders, respectively, are possible. However, these processes are degenerate in BF with the process $(0,-1) \rightarrow$ $(0,1)$ taking place without diffraction but just through the reflection of light by the plane compression/dilatation acoustic wave. The latter process takes place with the plane acoustic waves propagating along the $z$ direction, and is dominating over two other processes contributing to the same BF. Consequently, accounting for the processes $( \pm 1,-1) \rightarrow(\mp 1,1)$ and $( \pm 2,-1) \rightarrow(\mp 2,1)$ is not necessary for the explanation of our experimental observations. They are presented in Table I only for the sake of completeness. Thus, the experimental BFs from the upper part of Table I except $(-1,-1) \rightarrow(1,-1)$ can be explained by the processes involving photons of the zeroth and the first diffraction orders only. At the same time, the participation of the phonons from the second and even from the third diffraction orders [see, for example, $(-2,-1) \rightarrow$ $(1,-1)]$ is largely required in the processes with the photons from the second diffraction order in the lower part of Table I. It should be noted that the even order diffraction (second, forth,...) of the probe light as well as the acoustic waves is not prohibited because of the deviation of the ratio of the $\mathrm{Au}$ rod width to the period from $50 \%$.

To explain all the available experimental data, we do need to account for the phonon which is neither propagating nor decaying along the $z$ axis. The experimental frequencies of 20.6 and $25.0 \mathrm{GHz}$, observed only in the experiment with $\mathbf{E} \| \mathbf{y}$ polarization of the probe light in the $45^{\circ}$ and $60^{\circ}$ samples, respectively, can be currently associated only with the scattering of light by longitudinal phonons skimming along the sample surface. The corresponding scattering process is $(-1,-1) \rightarrow(1,-1)$.

The observation of the BFs only when using probe light polarized along the metallic rods, i.e., $\mathbf{E} \| \mathbf{y}$, is rather common to our experiments (see Fig. 4 and Table I). We attribute these observations to higher reflection/diffraction efficiency of $\mathbf{E} \| \mathbf{y}$ polarized light in comparison with the light polarized along the direction of grating periodicity, i.e., $\mathbf{E} \| \mathbf{x}$, both by metallic and acoustic gratings. On the one hand, this hypothesis is consistent with the known applications of metal gratings as birefringent light polarizers $[33,34]$. On the other hand, the scattering of $\mathbf{E} \| \mathbf{x}$ polarized light may have lower efficiency than that of $\mathbf{E} \| \mathbf{y}$ when the propagation direction of the scattered light is nearly perpendicular to the direction of the polarization, which is induced by the incident light and the acoustic waves [31,35]. It should be noted that this may have similar origin to Wood's anomaly for the diffraction efficiency of ordinary gratings.

Our experimental results confirm that the application of the diffraction grating in the TDBS experiments provides an opportunity to overcome some limitations of the TDBS technique discussed in Introduction. First, the proposed experimental scheme provides an opportunity to detect simultaneously multiple Brillouin frequencies from the highest possible BF in the backscattering configuration, $f_{B}^{\max }=44.22 \mathrm{GHz}$, to the lowest possible $\mathrm{BF}$ in the forward scattering configuration, $f_{B}^{\text {min }}=10.46 \mathrm{GHz}$ (see the first and the last lines in the upper part of Table I). This significantly broadens the frequency band of the TDBS from $f_{B}^{\min } \approx 0.7 f_{B}^{\max }$ (see Introduction) to $f_{B}^{\min } \approx 0.25 f_{B}^{\max }$. The highest BF detected in our experiments does not depend on the grating, while the lowest detected frequency is controlled by the grating period as it can be noticed from the diagrams in Fig. 6 where the several degenerate processes provide access to this frequency. It could be additionally diminished by increasing the period $p$ of the grating. The comparison of the lowest BFs detected in our two samples (first line in Table I) supports this expectation. Thus the frequency range accessed by TDBS could be additionally broadened by the dedicated preparation of the grating.

Our experiments and data analysis demonstrate that TDBS measurement in a single experimental configuration with diffraction grating, i.e., without any modification of the directions for optical pumping and probing of the sample, is sufficient to extract the refractive index $n$ and the sound velocity $v$ of the material. In principle, for this purpose, any two of the experimentally detected frequencies, which in Eq. (2) depend both on $n$ and $v$, could be used. It is also possible to determine the refractive index and sound speed by fitting simultaneously larger number the measured frequencies in order to increase statistically the reliability in their determination, if required [20]. This has been actually 
done for the calculation in Table I. Moreover, the detection of the BF corresponding to the forward scattering process of light by the skimming longitudinal wave, $(-1,-1) \rightarrow$ $(1,-1)$, provides opportunity to determine the sound velocity without knowledge of the refractive index. In this process $k_{B}=2 \pi f_{B} / v=2 q$ (see Fig. 6) and the determination of the sound velocity requires just the knowledge of the grating period, $v=p f_{B} / 2$.

Finally, it is worth mentioning that in our experiments the number of the detected forward scattering processes is smaller than the backward scattering ones (see Fig. 6). This is related to the fact that our scheme allows all possible backward scattering processes to be accessed in the second sequence of the probe light scattering from those described earlier. In this sequence in the acousto-optic interaction, both the light incident on the acoustic grating and the scattered probe light can be of the arbitrary diffractions orders, while in the third and the fourth scattering sequences for the detection of the forward scattering processes, only one of these light fields could be of an arbitrary diffraction order. This asymmetry in the TDBS scheme could be potentially corrected in the thinner experimental samples with two diffraction gratings deposited on the opposite sides when multiple reflections of the probe light between the surfaces of the sample are becoming important while the second grating (deposited on the back surface of the sample in Fig. 1) could, in particular, transmit in the detection direction in air the probe light incident on it from the sample side along an arbitrary order of diffraction. With the diffraction gratings on both sides of the sample all BS processes possible in the sample, both for forward and backward scattering, could potentially involve incident and scattered light in arbitrary diffraction orders.

\section{CONCLUSIONS}

We have performed picosecond acoustic interferometry [time-domain Brillouin scattering (TDBS)] measurements in transparent samples with metallic gratings. The pump light pulses absorbed in the metallic grating structure generate acoustic gratings (inhomogeneous plane compression/dilatation acoustic waves) in the substrate. The propagation of the acoustic gratings is monitored by delayed probe light pulses. By detecting the modulation of the probe light intensity in the first-order diffracted beam, we have observed in the time domain multiple Brillouin oscillations with rich frequency spectra. The obtained results are explained by a theoretical model which takes into account all possible configurations of the probe light scattering/diffraction by these acoustic gratings, including those where the light itself is diffracted by the metallic grating either before its scattering by the phonons or after the scattering, or both. The agreement between the experimental Brillouin frequencies and the calculated frequencies is fairly good.

Our theory revealed two reasons for the increased number of the BS processes that could be monitored in the scheme of TDBS proposed by us in comparison with the earlier reported experiments with metallic gratings [18]. Our scheme provided for the first time access by TDBS to both the forward and backward scattering processes of light by the coherent sound. In addition, one of our studied gratings provided propagating probe light in higher diffraction orders than in Ref. [18]. Access to both forward and backward scattering processes importantly broadens the range of frequencies accessible by TDBS. This fact in combination with the opportunities to monitor multiple different BS processes/frequencies simultaneously would be advantageous in studying the dispersion of the sound velocity and attenuation in many materials. It is worth noting here that the applications of usual TDBS schemes for studying acoustic wave attenuation are documented for a variety of the media [36-46]. The opportunity to monitor in a single measurement the acoustic phonons propagating in different directions could be attractive for revealing the elastic/inelastic anisotropy of materials, including one which could be caused by nonisotropic loading or by the residual stress. For the studies of the anisotropy, it is also extremely advantageous that gratings, as demonstrated by our experiments, can simultaneously launch phonons, detectable by TDBS in several discrete directions in the range from $0^{\circ}$ to $90^{\circ}$ relative to the sample surface. TDBS assisted by diffraction gratings could be also advantageous in several applications with limited optical access to the samples $[12,40,47]$. It could provide the opportunity to monitor multiple BS processes/frequencies even with incident and the detected/scattered light propagating collinearly (for example, in the case of the probe light incident normally on the grating and the detection of either reflected or transmitted light in the directions also normal to the grating surfaces). Another example along this line would be TDBS measurement of liquids contained in a transparent cell with appropriate grating patterns formed on the cell surface facing to the liquid.

However, we admit that our current experiments are not optimized for the above listed potential applications, because their goal was primarily just to confirm that multiple frequencies corresponding to both backward and forward Brillouin scattering processes can be detected simultaneously in a single optical scheme. For the potential applications, the experiments could be in the future accompanied by the application of the dedicated signal processing methods, providing increased visibility of the low amplitude Brillouin peaks. This could be achieved, for example, by filtering out the high-amplitude frequencies before the analysis of the low-amplitude peaks, when they are sufficiently separated either in the frequency domain or in the time domain. An improvement in the determination of the peak positions could be also potentially achieved by fitting all the peaks simultaneously in a multiparameter fit, as it is known for FDBS and x-ray diffraction spectra. In the experimental part, the quality of the experimental data could be improved by diminishing the velocity of the optical delay line and/or by using longer delay line in the current experiment scheme, which will lead to probing the system with better temporal/frequency resolution. Relative amplitudes of the different Brillouin peaks depend on the design of the metallic grating, in particular, on the metal filling factor of the grating. For example, the dedicated design could increase the amplitude of the peaks probed by the light scattered in the second diffraction order relative to the peaks probed by the light scattered in the first diffraction order, if necessary. In general, the increase of the peak amplitudes would lead to the opportunity to detect the corresponding frequencies above the noise level for longer time, and, thus, to narrow the width of 
the Brillouin peaks and make more precise their positioning, in the case, when noise and not the inherent attenuation of the acoustic waves is the main factor of line broadening. Potentially, the so-called death-star optical scheme for the pump pulse shaping $[41,48,49]$ could be also used for the selective enhancement of the particular Brillouin frequency, if necessary.

Finally, some of the functionalities of the proposed TDBS scheme could be achieved by replacing the metallic gratings by laser-induced gratings that could be generated by the interference pattern of two light beams propagating at an angle. Such gratings can launch acoustic gratings in the sample [28-30] and also diffract probe light [31], although much less efficiently than the metallic gratings. The advantage of laser gratings is in the perspective of noncontact and noninvasive diagnostics of the samples by TDBS. The drawback is in the more technically elaborated optical scheme.

\section{ACKNOWLEDGMENTS}

The reported research was conducted in the frame of the project PLUSDIL supported by ANR, under contract ANR-12BS09-0031. O.M. is partially supported by the Acoustic HUB of Région des Pays de la Loire in France, by a Grant-in-Aid for Scientific Research from Japan Society for the Promotion of Science (17H02807, 16K13713), and by a research grant from the Murata Science Foundation. We are grateful to Humphrey J. Maris for the comments. We would like to thank OPEN FACILITY (Hokkaido University Sousei Hall) for the sample fabrication.
[1] C. Thomsen, H. T. Grahn, H. J. Maris, and J. Tauc, Opt. Commun. 60, 55 (1986).

[2] H. T. Grahn, H. J. Maris, and J. Tauc, IEEE J. Quantum Electron. 25, 2562 (1989).

[3] O. B. Wright and T. Hyoguchi, Opt. Lett. 16, 1529 (1991).

[4] O. B. Wright, J. Appl. Phys. 71, 1617 (1992).

[5] K. E. O'Hara, X. Hu, and D. G. Cahill, J. Appl. Phys. 90, 4852 (2001).

[6] A. Devos, R. Côte, G. Caruyer, and A. Lefévre, Appl. Phys. Lett. 86, 211903 (2005).

[7] C. Mechri, P. Ruello, J. M. Breteau, M. R. Baklanov, P. Verdonck, and V. Gusev, Appl. Phys. Lett. 95, 091907 (2009).

[8] A. Steigerwald, Y. Xu, J. Qi, J. Gregory, X. Liu, J. K. Furdyna, K. Varga, A. B. Hmelo, G. Lüpke, L. C. Feldman et al., Appl. Phys. Lett. 94, 111910 (2009).

[9] D. Yarotski, E. F. L. Yan, Q. Jia, Y. Wang, A. J. Taylor, and B. P. Uberuaga, Appl. Phys. Lett. 100, 251603 (2012).

[10] T. Dehoux, N. Tsapis, and B. Audoin, Soft. Matter. 8, 2586 (2012).

[11] I.-J. Chen, P.-A. Mante, C.-K. Chang, S.-C. Yang, H.-Y. Chen, Y.-R. Huang, L.-C. Chen, K.-H. Chen, V. Gusev, and C.-K. Sun, Nano Lett. 14, 1317 (2014).

[12] S. M. Nikitin, N. Chigarev, V. Tournat, A. Bulou, D. Gasteau, B. Castagnede, A. Zerr, and V. E. Gusev1, Sci. Rep. 5, 9352 (2015).

[13] T. Dehoux, K. Ishikawa, P. H. Otsuka, M. Tomoda, O. Matsuda, M. Fujiwara, S. Takeuchi, I. A. Veres, V. E. Gusev, and O. B. Wright, Light Sci. Appl. 5, e16082 (2016).

[14] I. L. Fabelinskii, Molecular Scattering of Light (Plenum, New York, 1968).

[15] W. Hayes and R. Loudon, Scattering of Light by Crystals (Wiley, New York, 1978).

[16] J. G. Dil, Rep. Prog. Phys. 45, 285 (1982).

[17] A. Polian, J. Raman Spectrosc. 34, 633 (2003).

[18] H. N. Lin, H. J. Maris, L. B. Freund, K. Y. Lee, H. Luhn, and D. P. Kem, J. Appl. Phys. 73, 37 (1993).

[19] R. Côte and A. Devos, Rev. Sci. Instrum. 76, 053906 (2005).

[20] A. M. Lomonosov, A. Ayouch, P. Ruello, G. Vaudel, M. R. Baklanov, P. Verdonck, L. Zhao, and V. E. Gusev, ACS Nano 6, 1410 (2012).
[21] M. Tomoda, O. Matsuda, and O. B. Wright, Appl. Phys. Lett. 90, 041114 (2007).

[22] H. Eichler and H. Stahl, J. Appl. Phys. 44, 3429 (1973).

[23] K. A. Nelson, R. J. D. Miller, D. R. Lutz, and M. D. Fayer, J. Appl. Phys. 53, 1144 (1986).

[24] M. D. Fayer, IEEE J. Quantum Electron. 22, 1437 (1986).

[25] A. A. Maznev, D. J. McAulifee, A. G. Doukas, and K. A. Nelson, Ultrasound Med. Biol. 25, 601 (1999).

[26] B. C. Daly, N. C. R. Holme, T. Buma, C. Branciard,, T. B. Norris, D. M. Tennant, J. A. Taylor, J. E. Bower, and S. Pau, Appl. Phys. Lett. 84, 5180 (2004).

[27] C. Jean, L. Belliard, T. W. Cornelius, O. Thomas, Y. Pennec, M. Cassinelli, M. E. Toimil-Molares,, and B. Perrin, Nano Lett. 16, 6592 (2016).

[28] V. Gusev, Appl. Phys. Lett. 94, 164105 (2009).

[29] V. Gusev, J. Appl. Phys. 107, 114906 (2010).

[30] M. Kouyate, T. Pezeril, D. Mounier, and V. Gusev, J. Appl. Phys. 110, 123526 (2011).

[31] M. Kouyaté, T. Pezeril, V. Gusev, and O. Matsuda, J. Opt. Soc. Am. B 33, 2634 (2016).

[32] CRC Handbook of Chemistry and Physics, edited by D. R. Lide, 84th ed. (CRC Press, Boca Raton, Fla., 2003).

[33] L. Zhou and W. Liu, Opt. Lett. 30, 1434 (2005).

[34] D. Zhang, P. Wang, X. Jiao, C. Min, G. Yuan, Y. Deng, H. Ming, L. Zhang, and W. Liu, Appl. Phys. B 85, 139 (2006).

[35] M. Born and E. Wolf, Principles of Optics, 7th ed. (Cambridge University Press, UK, 1999).

[36] H. N. Lin, R. J. Stoner, H. J. Maris, and J. Tauc, J. Appl. Phys. 69, 3816 (1991).

[37] W. Chen, H. J. Maris, Z. R. Wasilewski, and S. Tamura, Philos. Mag. B 70, 687 (1994).

[38] P. Emery and A. Devos, Appl. Phys. Lett. 89, 191904 (2006).

[39] A. Devos, M. Foret, S. Ayrinhac, P. Emery, and B. Rufflé, Phys. Rev. B 77, 100201 (2008).

[40] F. Decremps, L. Belliard, B. Perrin, and M. Gauthier, Phys. Rev. Lett. 100, 035502 (2008).

[41] T. Pezeril, C. Klieber, S. Andrieu, and K. A. Nelson, Phys. Rev. Lett. 102, 107402 (2009). 
[42] S. Ayrinhac, M. Foret, A. Devos, B. Rufflé, E. Courtens, and R. Vacher, Phys. Rev. B 83, 014204 (2011).

[43] A. A. Maznev, K. J. Manke, C. Klieber, K. A. Nelson, S. H. Baek, and C. B. Eom, Opt. Lett. 36, 2925 (2011).

[44] C. Klieber, T. Hecksher, T. Pezeril, D. H. Torchinsky, J. C. Dyre, and K. A. Nelson, J. Chem. Phys. 138, 12A544 (2013).

[45] A. Maehara, N. Nakamura, H. Ogi, and M. Hirao, Jpn. J. Appl. Phys. 53, 086602 (2014).

[46] S. Danworaphong, M. Tomoda, Y. Matsumoto, O. Matsuda, T. Ohashi, H. Watanabe, M. Nagayama, K. Gohara, P. H. Otsuka, and O. B. Wright, Appl. Phys. Lett. 106, 163701 (2015).
[47] M. Kuriakose, S. Raetz, N. Chigarev, S. M. Nikitin, A. Bulou, D. Gasteau, V. Tournat, B. Castagnede, A. Zerr, and V. E. Gusev, Ultrasonics 69, 259 (2016).

[48] J. D. Beers, M. Yamaguchi, T. Feurer, B. J. Paxton, and K. A. Nelson, in Ultrafast Phenomena XIV, edited by T. obayashi, T. Okada, T. Kobayashi, K. A. Nelson, and S. de Silvestri, Springer Series of Chemical Physics Vol. 79 (Splinger, Berlin, Heiderberg, 2005), pp. 236-238.

[49] C. Klieber, E. Peronne, K. Katayama, J. Choi, M. Yamaguchi, T. Pezeril, and K. A. Nelson, Appl. Phys. Lett. 98, 211908 (2011). 